\title{
ESPRIT DE LIBERTÉ ET ENRACINEMENT HONGROIS DANS LA CRÉATION LISZTIENNE
}

\author{
BRUNO MOYSAN \\ Agrégé, docteur en musicologie, chargé d'enseignement à l'Université de Versailles \\ brunomoysan@aol.com
}

\begin{abstract}
Liszt fut un génie de son temps autant qu'un génie de la musique. Restant inclassable, malgré son tempérament romantique, il inventa le format du récital qui lui permettait de se donner en spectacle, seul et à travers toute l'Europe, sans reposer le bon vouloir d'un mécène. Sa recherche effrénée de liberté coïncide, en outre, avec l'esprit qui soufflait alors sur la Hongrie, qui allait culminer avec la révolution de 1848. C'est ainsi que la liberté lisztienne trouve son enracinement dans un territoire, une patrie, qui restèrent par ailleurs chez lui largement fantasmés.
\end{abstract}

Mots-clefs : Liszt, liberté, enracinement, $\mathrm{XIX}^{\mathrm{e}}$ siècle, sujet moderne, récital

La pensée contemporaine tend à faire de l'enracinement et de la liberté deux principes antinomiques. Toute limite, tout point de fixation est considéré comme limitatif de la liberté infinie des modernes. Dès lors, il n'est rien de plus logique que la nation, avec ce qu'elle présuppose de limitation et d'auto-limitation, tout simplement parce qu'elle fixe des frontières et définit des identités, soit l'objet d'une hostilité à la mesure de la propension des sociétés modernes, ou plutôt post-modernes, d'ailleurs, à devenir liquides ${ }^{1}$. Au XIX ${ }^{\mathrm{e}}$ siècle, il n'en était pas ainsi. Au lieu d'être perçue comme limitative de l'individu, la nation est alors, au contraire, une promesse d'auto-détermination et de liberté, cela contre la dimension oppressive et inclusive des grands empires, contre les logiques assimilatrices par acculturation ascendante, profondément élitistes, de la société de cour cosmopolite des XVII ${ }^{e}$ et XVIII ${ }^{e}$ siècles. L'internationalisme civilisateur des élites curiales de l'absolutisme assujettit l'artiste à un réseau de contraintes que l'individualisme romantique se refusera à pérenniser. Aussi n'est-il pas étonnant que Liszt ait vu dans l'éveil de la nation hongroise une dynamique de libération en harmonie avec ses propres ambitions émancipatrices, que ces dernières soient d'ordre esthétique ou social. Liberté artistique et éveil national mettent au premier plan l'individu en tant qu'acteur-stratège dans la mesure où, même si l'on soutient d'ordinaire que la modernité a consacré l'avènement des masses, ce sont bien les individus d'exception qui ont permis aux masses de prendre conscience d'elles-mêmes et d'être ainsi actrices de leur propre destin. Mais laissons justement la place à notre acteur-stratège. 


\section{Liszt et l'esprit de liberté}

Liszt a chèrement payé le prix de la liberté : scandales, anathèmes, moqueries, solitude, incompréhension, indifférence. Même au plus fort de ses succès de pianiste, pendant cette période virtuose qu'on a appelée la Glanz-Period, la remise en grande pompe du fameux sabre d'honneur, à Budapest, en 1841, qui le sacrait héros de la cause nationale hongroise, lui a aussi valu le quatrain ${ }^{2}$ suivant :

Parmi tous ces guerriers, Liszt seul est sans reproche, Car, malgré son grand sabre, on sait que ce héros,

N'a vaincu que des doubles croches

Et tué que des pianos!

Beaucoup plus tard, il écrira à son ami Odon Mihailovitch : « Tout le monde est contre moi. Catholiques, car ils trouvent ma musique d'église profane, protestants car pour eux ma musique est catholique, franc-maçons car ils sentent ma musique cléricale ; pour les conservateurs, je suis un révolutionnaire, pour les aveniristes un faux jacobin. Quant aux Italiens, malgré Sgambati, s'ils sont garibaldiens, ils me détestent comme cagot, s'ils sont côté Vatican, on m'accuse de transporter la grotte de Vénus dans l'église. Pour Bayreuth, je ne suis pas un compositeur, mais un agent publicitaire. Les Allemands répugnent à ma musique comme française, les Français comme allemande, pour les Autrichiens, je fais de la musique tzigane, pour les Hongrois, de la musique étrangère ${ }^{3}$.» Tout en s'efforçant vainement de s'adapter, Liszt n'était pas véritablement plastique à son environnement. Nul n'aura plus que lui, en définitive, incarné l'esprit de liberté des modernes, mais un esprit de liberté un peu composite où l'on retrouve, en reprenant la célèbre distinction de Tocqueville, à la fois des éléments de liberté aristocratique et de liberté démocratique : "Cette notion aristocratique de la liberté produit chez ceux qui l'ont reçue un sentiment exalté de leur valeur individuelle et un goût passionné pour l'indépendance. Elle donne à l'égoösme une énergie et une puissance singulière. Conçue par des individus, elle a souvent porté les hommes aux actions les plus extraordinaires ; adoptée par une nation tout entière elle a créé les plus grands peuples qui fussent jamais. [...] D'après la notion moderne, la notion démocratique, et j'ose dire la notion juste de la liberté, chaque homme étant présumé avoir reçu de la nature les lumières nécessaires pour se conduire, apporte en naissant un droit égal et imprescriptible à vivre indépendant de ses semblables, en tout ce qui n'a rapport qu'à lui-même, et à régler comme il l'entend sa propre destinée. [...] Chacun ayant un droit absolu sur lui-même, il en résulte que la volonté souveraine ne peut émaner que de l'union de la volonté de tous ${ }^{4}$. »

L'une des particularités de Liszt est l'unité profonde de sa vie, en dépit d'un apparent émiettement. Virtuose, compositeur, écrivain, penseur, il sillonne l'Eu- 
rope et s'intéresse à tout : musique, philosophie, peinture, architecture, théorie politique, théologie. Né à la limite de la Hongrie et de l'actuelle Autriche sur les terres des Esterhazy, les mécènes de Haydn, il étudie à Vienne avec Czerny, l'unique élève de Beethoven, devient une des personnalités les plus en vue du monde parisien romantique, sillonne l'Europe, se fixe à Weimar puis à Rome et pour finir erre presque vingt ans entre Weimar, Rome et Budapest, séjournant à peu près trois mois par an successivement dans ces trois villes. Chez ce personnage singulier, transgressif, qui ose vivre maritalement avec une comtesse, puis avec une princesse, et qui finit, à l'étonnement de tout le monde, tertiaire franciscain et nomade, les manifestations apparemment hétérogènes de la liberté semblent converger dans ce que Paul Bénichou a appelé « le sacerdoce de l'art $»^{5}$. Le renversement du rapport de sujétion entre l'artiste et son environnement est la première manifestation, musicale, de cette liberté artistique émancipatrice qui aura été le grand combat de Liszt. En devenant à la fois virtuose-compositeur et compositeur-virtuose, Liszt renonce à un emploi typique de la société de cour absolutiste, celui de compositeur de cour. Cet emploi parfaitement normé, occupé par Lully à Versailles, Haydn à Eisenstadt ou Mozart à Salzbourg, était sécurisant, mais il était non moins assujettissant. En y renonçant, Liszt se trouve dans une situation beaucoup plus libre, mais avec pour prix à payer une forte marge d'incertitude et d'imprévisibilité en même temps que l'obligation de se soumettre, cette fois-ci, aux lois de la concurrence et du marché. Au système de négociation à dominante bi-polaire, "artiste-mécène" (qui n’interdit pas les négociations avec d'autres types de partenaires comme les éditeurs, par exemple, ou encore la presse), fait place un système de négociations franchement multipolaire : artiste, public, presse, éditeurs, pouvoir politique, entrepreneurs de spectacles...

Le plus frappant, chez Liszt, est la façon dont il dessine un chemin de liberté à travers les contraintes du Paris romantique de la monarchie de Juillet, puis d'une Europe travaillée par les idées nouvelles, mais qui reste encore une Europe des cours. Dans un premier temps, avant 1848, Liszt sépare nettement le répertoire de concert et les compositions éditées. Au public de ses concerts à Paris et en Europe : les fantaisies sur des thèmes d'opéras à la mode et le Grand galop chromatique. Aux grands éditeurs du temps comme Schlesinger ou Breitkopf : 1'Album d'un voyageur, les Grandes études, les Harmonies poétiques et religieuses, monuments destinés implicitement à la postérité mais qu'il se garde bien de jouer en concert. Liszt n'en est pas pour autant schizophrène, car l'une de ses stratégies les plus subtiles, comme Chopin d'ailleurs, par exemple dans ses Valses, est de manipuler les codes d'un style mondain aisément accessible tout en donnant à ces mêmes codes un contenu nouveau. Là réside toute l'originalité de ses fantaisies sur des thèmes d'opéras, comme les Réminiscences de Lucrezia Borgia ou encore les Réminiscences des Huguenots, qui est de s'inscrire dans l'événement, dans le brouhaha de la mode et du succès, dans l'onde de choc d'un grand genre 
comme l'opéra (a contrario la fantaisie pour piano est un petit genre), mais de proposer dans le même temps une modernité de forme et de langage qui n'a rien à envier à celle des œuvres les plus révolutionnaires de Chopin ou de Schumann. À ce titre, Liszt est un créateur véritablement subversif ${ }^{6}$, car il donne à entendre autre chose que les codes dominant, mais d'une manière qui semble au premier abord simplement revisitées, alors qu'elle est transformée en profondeur. Dans ce qui apparaît comme une sorte de fausse soumission aux codes de la mondanité historiquement imposés par les élites aristocratiques, Liszt propose des œuvres de concert au style mondain, romantique, aptes à véhiculer les revendications les plus libertaires de l'artiste moderne.

La particularité de Liszt est d'associer ce mécanisme subversif, portant sur le style et le langage musical, à la pratique sociale elle aussi nouvelle qu'est le concert soliste. Liszt est en effet le premier à se présenter seul devant un public, inaugurant la forme que l'on désignera désormais comme un récital. C'est une véritable révolution, car avant lui le concert était éclectique, hétérogène, panaché - peu importe la terminologie -, c'est-à-dire que dans la même soirée se côtoyaient divers musiciens (instrumentistes, chanteurs, formations de chambre, orchestre) chargés de donner un programme diversifié. Liszt, tel Victor Hugo prophétisant de son rocher de Guernesey, invente la structure de sociabilité musicale qui correspond, d'une part, à la souveraineté narcissique de l'individu moderne, d'autre part, aux revendications de l'artiste romantique à détenir un pouvoir spirituel laïque au sein d'une société en voie de démocratisation et de sécularisation. Après 1848, Liszt fera de Weimar, la ville de Goethe et de Schiller, son rocher de Guernesey et le lieu de son apostolat esthétique. Il y créera, à a tête de l'orchestre de la ville, les plus grandes œuvres de ses contemporains, Wagner, Verdi, Berlioz, tout en en faisant le lieu où il composera aussi ses plus grandes œuvres, Sonate en si mineur, Poèmes symphoniques. Pèlerin infatigable, il tentera de faire de Rome le lieu d'une musique d'Église renouvelée. Mais toujours libre de toute sujétion, que ce soit celle des princes, du pape ou du conformisme ambiant. Toujours obsédée par l'avenir, la liberté artistique lisztienne est, en définitive, à la fois d'essence aristocratique et d'essence démocratique. De la liberté aristocratique, elle a, d'une certaine manière, dans la lignée d'un Chateaubriand que Liszt admirait profondément, le « sentiment exalté de 1[a] valeur individuelle et un goût passionné pour l'indépendance $»^{7}$. Elle puise dans les réserves narcissiques du Moi romantique cet « égoïsme » qui, pour Tocqueville, donne à l'individu cette « énergie » et cette " puissance singulière » qui « porte [...] aux actions les plus extraordinaires ». De la liberté démocratique, elle a cette propension à l'autonomie qui « apporte en naissant [à l'individu] un droit égal et imprescriptible à vivre indépendant de ses semblables, en tout ce qui n'a rapport qu'à lui-même, et à régler comme il l'entend sa propre destinée. [...] » Par un curieux mécanisme de réappropriation et de retournement, l'individualisme lisztien, comme celui des 
romantiques en général, qui est celui d'un « musicien bourgeois $»^{8}$ dans une société encore profondément aristocratique, mais déjà travaillée par la démocratie naissante, reprend les traits essentiels de la liberté aristocratique et les retourne contre l'héritage de l'Ancien Régime, cela pour les mettre au service de la définition d'un élitisme nouveau, typiquement moderne, car fondé sur le Moi ainsi que sur les mérites de l'individu autonome et créateur, y compris de lui-même. Mais cette liberté - et c'est en cela qu'elle se différencie d'une liberté purement aristocratique dont, par exemple, un Chateaubriand pouvait s'enorgueillir-, cette liberté est profondément insérée dans le collectif propre à la démocratie moderne, dans la mesure où « chacun ayant un droit absolu sur lui-même, il en résulte que la volonté souveraine ne peut émaner que de l'union de la volonté de tous $»^{9}$. C'est dans ce dernier trait que prend sa source la dimension proprement engagée d'un humanitarisme artistique et progressiste proprement lisztien, qui ne sépare aucunement l'art en tant que tel de la justice sociale, de la lutte contre les inégalités, du combat pour une civilisation meilleure.

\section{La Hongrie}

En se disant lui-même « tzigane et franciscain », Liszt associe une errance-désir (le tzigane et le frère mineur ne sont-ils pas tout deux des errants ?) à deux notions, ou objets, celle de Hongrie et celle de Dieu. Paradoxalement, la relation à la patrie et la relation à Dieu sont strictement renversées. La patrie, dans le cas de Liszt l'exilé, est le lieu d'où l'on vient, le lieu des nostalgies fabriquées à partir desquelles se définit l'errance du présent. Dieu, au contraire, est le lieu où l'on va dans une relation qui vous échappe (《Deus absconditus », «Dieu caché ») au fur et à mesure qu'elle se tisse («Console-toi, tu ne me chercherais pas si tu ne m'avais trouvé » dit Dieu dans les Pensées de Pascal), vous appauvrit de vous-même au fur et à mesure qu'elle vous enrichit. Chez Liszt, la relation désir/ objet est une quête d'identité. Avant 1848, ses pratiques mondaines et son humanitarisme associaient son image du Moi à une combinaison d'élitisme et d'universalité sociale ; le sacerdoce poétique de Weimar assimile ainsi le Moi à une production : l'œuvre dans l'histoire. Avec la Hongrie et Dieu, Liszt identifie le Moi à la vacuité en unissant par l'errance et la pauvreté (la tziganité, la mendicité du poverello) le lieu d'où l'on vient (la Terre) et le lieu où l'on va (Dieu).

L'engagement de Liszt est incontestable, bien qu'il soit né dans une famille germanophone à l'extrême ouest des limites de l'ancien royaume de Hongrie et n'ait jamais véritablement maîtrisé la langue hongroise. La révolution de 1848 lui donne ce que celle de 1830 a donné à Chopin : un moyen de s'identifier à la revendication d'un peuple exigeant le droit à disposer de lui-même, ce droit étant corrélé à l'autre passion typiquement moderne qu'est celle de la liberté. Liberté 
politique et liberté artistique sont ici étroitement liées. En retour, dans le contexte de la construction d'une autre mythologie elle aussi typiquement moderne, celle de l'artiste lui-même, le musicien gagne la possibilité de se constituer en symbole national. Or, en 1830, la question polonaise occupe tout l'espace, au grand profit de Chopin, il n'y a pas de question hongroise en 1830 à laquelle Liszt peut s'identifier. Mais son génie aura été d'avoir su préparer 1848 avant 1848. En 1839, une catastrophe naturelle ayant provoqué un drame humanitaire vient lui apporter comme sur un plateau ce que le politique stricto sensu tarde à lui procurer : ce sont les fameuses inondations du Danube à Pest. Lorsque le musicien revient dans son pays natal, il est auréolé du prestige que seul peut avoir un artiste qui a conquis Paris. Aussi ne sera-t-il pas étonnant de le voir reçu de manière princière par les magnats hongrois qui ont déjà été les mécènes de sa jeune carrière. On le reçoit triomphalement à Pest, lui le fils du modeste intendant des Esterhazy, après qu'il a fait le voyage depuis Presbourg (Pozsony) dans le coupé du comte Casimir Esterházy. Liszt donnera neuf concerts à Buda et Pest et y fera entendre sa fameuse Marche de Rákóczy. C'est à l'occasion de l'un de ces concerts, celui du 4 janvier 1840, qu'il reçoit des mains du comte Festetics et de quelques autres membres de l'aristocratie hongroise (comte Teleki, barons Bánffy et Augusz, Rudolf von Eckstein et Paul Nyáry), le sabre d'honneur dont il a été question plus haut, brillant de joyaux et dont on dit qu'il portait sur le fourreau l'inscription suivante : "À Franz Liszt, au grand artiste en reconnaissance de ses mérites artistiques et de son fervent amour de la patrie ».

Il convient de souligner que la série de concerts en faveur des inondés de Pest et la remise du sabre sont encadrés par une série de concerts donnés à Vienne, dont un à la Cour, le 29 novembre, et un au Théâtre de la Cour, le 4 février. Liszt montre en effet un sens très subtil de l'équilibre entre Budapest et Vienne. Quel pouvoir peut s'opposer à la compassion envers un drame humanitaire, même si ce dernier se colore de subversion politique ? Et qui peut s'opposer à l'ancien élève de Czerny et Salieri, lancé à 1823 à Paris muni de deux lettres de recommandations signées par Metternich lui-même et de retour au pays après non seulement la consécration parisienne, mais aussi un triomphe en Italie? Le 2 février, alors qu'il est de nouveau à Vienne, Liszt joue en concert ses premiers Magyar Dalok (Mélodies hongroises). La marche de Rákóczy jouée à Budapest n'a jamais été très en cour à Vienne et faire entendre au théâtre de la cour des Magyar Dalok n'est pas sans revêtir une dimension provocatrice analogue à celle que pouvait avoir une Polonaise de Chopin. Du côté des élites hongroises, offrir à un artiste (un saltimbanque) un sabre d'honneur (symbole militaire en principe réservé aux aristocrates) est véritablement donner à la musique et au musicien un rôle politique. Pour les magnats hongrois, récupérer, au profit de la cause nationale hongroise, un artiste aussi prestigieux et aussi parisien que Liszt était une fantastique opération subversive, cela neuf ans avant les événements de 1848, vingt sept ans avant la double monarchie. 
Les Magyar Dalok ont été composés dans l'émotion du retour aux sources. Il s'agit d'une série de onze pièces de courtes durée que le musicien avait conçue au départ comme étant le troisième cahier de l'Album d'un voyageur. On sait que, pour lui, musique hongroise et musique tzigane tendent à se confondre - ce qui finalement est de peu d'importance, car seule compte, à l'époque de l'éveil des nationalités, la référence nationale suscitée par la couleur locale du langage.

Ce premier cahier de Magyar Dalok est dédié à des personnalités aristocratiques engagées de près ou de loin dans la cause nationale hongroise : le comte Leo Festetics ( $\left.\mathrm{n}^{\circ} 1-6\right)$, le comte Casimir Esterházy ( ${ }^{\circ}$ 7-9) et le comte Alberti $\left(n^{\circ} 9-11\right)$. Le recueil est publié, ce qui en soi est un acte politique de provocation envers l'Empereur, à Vienne. Les Magyar Dalok, tout comme les recueils qui vont suivre (des Magyar Rapsodiak en 1846 puis, l'année suivante, le Carnaval de Pest) ont non seulement une portée politique, mais aussi une dimension nouvelle du point de vue esthétique. La référence hongroise s'inscrit dans une démarche romantique qui est celle de la recherche et de l'expression de la liberté. Comme Liszt l'écrira plus tard au jeune Tzigane Josi : « J'en viendrais presque à vous envier d'avoir échappé à l'art civilisé de faire de la musique, avec ses limites et restrictions... Aucun bavardage, ni jargon de pédants, chicaneurs, critiques et engeance sans nom de la même espèce ne peut vous atteindre ; de l'archet de votre violon, vous vous élevez au dessus de toute la misère du monde, et continuez à jouer d'un air de défi. » À ce titre, la Hongrie est pour Liszt ce que la Suisse était peut-être pour Rousseau ou Sénancour, dans sa recherche du ressourcement, mais d'un ressourcement qui, en raison du caractère particulier de la musique tzigane, permettrait au musicien d'être provocateur et politique, homme d'action, et donc... virtuose au sens propre et étymologique du mot. Le mythe d'une création épargnée par les corruptions multiples de la civilisation permet au poète, nourri d'Ossian et des $E d d a$, d'inventer les origines fabuleuses d'une antique épopée qui ne serait qu'instrumentale. À la lumière de sa volonté d'être le rhapsode d'une "épopée bohémienne" 10 dont l'histoire n'aurait laissé que des lambeaux, il faut interpréter le changement de désignation qui, en 1846, conduit Liszt à remplacer le titre Magyar Dalok par Magyar Rapsodiak - Rhapsodies hongroises. Le nationalisme devient mythe des origines. Cet enracinement de l'idée de nation dans une culture primitive, au sens rousseauiste du terme, a pour conséquence un travail spécifique de composition. Comme l'écrit Liszt lui-même dans Des Bohémiens et de leur musique en Hongrie : "Alors nous acquîmes la conviction que ces morceaux détachés, ces mélodies disjointes et éparses, étaient les parties disséminées, éparpillées, émiettées d'un grand tout ; qu'elles se prêtaient parfaitement à la construction d'un ensemble harmonieux, qui renfermerait la quintessence de leurs qualités les plus marquantes, le résumé de leurs beautés les plus frappantes, pouvant être considéré comme une sorte d'épopée nationale ; - épopée bohémienne - chantée dans une langue et dans une forme inusitées, 
comme est inusité tout ce que fait le peuple qui l'a créée ${ }^{11}$. » Liszt applique à la nature hongroise un type de rapport à l'emprunt et à la réécriture qu'il avait jusqu'alors pratiqué dans le domaine de la culture, avec, notamment, ses fantaisies sur des thèmes d'opéra ${ }^{12}$. La Sixième Rhapsodie, célèbre entre toutes, est un bon exemple du travail du rhapsode. Elle a connu plusieurs versions antérieures. On trouve des fragments de ses différentes sections dans les $n^{\circ} 4-5$ et 11 du premier cahier des Magyar Dalok. En 1844, Liszt publie chez Bernard Latte un petit volume intitulé Mélodies hongroises / Album d'un voyageur 3e année où l'on trouve une ébauche déjà plus élaborée de ce qui sera l'œuvre dans son acception définitive : tempo giusto en ré bémol majeur $\left(\mathrm{n}^{\circ} 1\right)$, Animato quasi presto en ut dièse majeur $\left(n^{\circ} 2\right)$, Allegretto en si bémol majeur $\left(n^{\circ} 3\right)$. Entre le $n^{\circ} 2$ et le $n^{\circ} 3$, Liszt compose un Preludio de 19 mesures, sorte de raccord permettant d'annoncer les éléments essentiels du n ${ }^{\circ} 3$ qui va suivre : la tonalité de si bémol majeur, le rythme caractéristique de syncope et l'enchaînement si majeur/si bémol majeur. C'est ce prélude qui sera remplacé par le magnifique Andante quasi improvisato de la version définitive, lui-même issu du Lento a capriccio malinconico de 1'Ungarische Rapsodien $\mathrm{n}^{\circ} 20$ de 1846. La Sixième Rhapsodie hongroise dans sa version définitive n'est que la réécriture d'une mosaïque de fragments dans une forme longue, rhapsodique.

Lors des événements de 1848, Liszt, fait un bref séjour à Vienne en mai, puis... brille par son absence. Après un petit tour sur les barricades, il évite soigneusement Vienne au plus fort de la crise, préférant Eisenstadt et Raiding. À la mi-juin 1849, au moment où les armées du tsar entrent en Hongrie, il est à Weimar. Un peu plus tard, au moment de la terrible répression d'octobre, il est encore à Weimar. Certes, il compose Funérailles, mais, en octobre 1849, c'est aussi la mort de Chopin... Là encore, Liszt fait preuve de subtilité en évitant de heurter de front le pouvoir central viennois.

Les quinze Rhapsodies hongroises éditées en $1851\left(\mathrm{n}^{\circ} 1\right.$ et 2$)$ et $1853\left(\mathrm{n}^{\circ} 3-15\right)$ constituent le stade ultime de cette évolution de l'esthétique du fragment vers celle de la forme longue, évolution qui a pour origine la prise de conscience progressive d'une mission, où l'expression artistique de l'universel ne peut être pleinement politique que si elle est poétique. Ces Rhapsodies sont contemporaines de la Sonate en si mineur, de la Deuxième Ballade. Un tel voisinage et l'ambition poétique dont témoigne Des Bohémiens et de leur musique en Hongrie oblige à réévaluer leur importance. Comme dans la Sonate en si mineur la virtuosité doit être sublimée dans une poétique.

Tardivement, entre 1882 et 1885, Liszt ajoutera quatre autres Rhapsodies. Le compositeur ne s'écarte pas de l'esthétique générale qui est celle des œuvres de sa dernière période. La surabondance virtuose devient étrange, décantée. La même année 1885 voit la composition des sept Historische Ungarische Bildnisse (Portraits nationaux hongrois), sombres évocations des figures de l'indépendance 
hongroise : István Széchenyi, Sándor Petöfi, László Teleki... Le voisinage de ces noms symboliques avec les quatre dernières rhapsodies atteste de la permanence des événements de 1839 dans la mémoire lisztienne et éclairent l'épopée mythique du cahier de 1851-1853. Chez le grand musicien, le politique s'incarne dans une nation qui, elle même, s'identifie à un dépôt mythique et légendaire élaboré, déposé, transmis et fragmenté anonymement au cours des siècles. Liszt, dans ses ultimes productions hongroises, réduit à l'élémentaire les oeuvres qu'il avait conçues et achevée au cours des années précédentes. L'invention du folklore à partir de la mémoire reste ici une exploration identitaire, mais d'une identité dépouillée de toute préoccupation constructiviste, de cette préoccupation constructiviste et militante qui était celle de la période de Weimar. Le nationalisme de 1839-67 et de l'épopée rhapsodique du cahier de 1851-53 cèdent le pas à des œuvres déconstruites, obsédées par le sentiment de la mort; sans oublier une prolifération d'éléments hongrois dans des pièces a priori non hongroises comme la Troisième Année de Pèlerinage ou la Troisième Ode funèbre.

La Hongrie serait-elle aussi devenue une vanité, une attache inutile?

\section{Conclusion}

Dans la dernière partie de sa vie, à partir de 1869, Liszt nomadise entre Budapest, Weimar et Rome. C'est avant tout par sa subjectivité que le grand virtuose, toujours en mouvement, assure la synthèse de ces différents ancrages qui, par bien des côtés, sont antinomiques. En effet, Weimar la protestante s'oppose à Rome la catholique et Budapest la hongroise revendique une langue qui s'oppose à l'allemand de l'Empire et un pluralisme religieux qui n'est ni celui de Rome ni celui de Weimar. La liberté de Liszt est bien sûr celle de la modernité romantique. Elle est avant tout un regard, fondamentalement de l'ordre de la lecture-interprétation. En ce sens le regard de Liszt sur son environnement, aussi bien sur la société de son temps que sur les matériaux hétérogènes qu'il unifie dans l'acte de composition (d'une fantaisie, par exemple sur un thème d'opéra, ou d'une rhapsodie hongroise), voire sur lui-même relève de ce que Marcel Gauchet, commentant les historiens romantiques des années 1820-30, désigne comme un travail de recomposition seconde ${ }^{13}$ en ce qu'il conduit, étant l'œuvre du sujet autonome, à une action transformatrice sur le monde lu et interprété par le sujet interprétant et agissant.

Cette mise au premier plan de la subjectivité autonome, c'est-à-dire libre de toute forme d'hétéronomie et en même temps transformatrice de l'existant, a aussi pour conséquence une redéfinition de la notion d'aristocratie. L'engagement de Liszt dans la cité, y compris sous son aspect de collectivité nationale à l'auto-définition de laquelle il participe activement, et son combat pour le progrès en art, 
plus qu'ils ne rompent avec l'aristocratie traditionnelle en sont plutôt la mutation. La modernité n'a pas en effet supprimé l'aristocratie, elle a simplement modifié son rapport au temps. Saint-Simon, dans ses Lettres d'un habitant de Genève à ses contemporains, propose une aristocratie nouvelle qui tire sa capacité à diriger l'opinion publique non parce qu'elle incarne une permanence, mais parce qu'elle est en mesure de voir ce que les autres ne voient pas ou mal, c'est-à-dire là où doit aller l'humanité, autrement dit : l'avenir, puisqu'elle regarde « avec l'œil du génie la situation actuelle de l'esprit humain ». "Savants, artistes, écrit Saint-Simon, regardez avec l'œil du génie la situation actuelle de l'esprit humain ; vous verrez que le sceptre de l'opinion publique est entré dans vos mains; saisissez-le donc vigoureusement ${ }^{14}$. » La flèche du Temps s'est inversée : de permanence du passé dans le présent, l'aristocratie moderne devient une vision du futur dans le présent, d'où sa propension à mettre en scène une posture prophétique. Le voyant, comme Liszt, est celui qui effectue les synthèses opérationnelles susceptibles d'agir sur la collectivité et de lui donner une direction.

\section{Notes}

1 Sur la notion de société liquide et de modernité liquide, voir bien sûr, entre autre : Zygmunt Bauman, Le présent liquide, Paris, Seuil, 2007

2 Cité dans Serge Gut, Liszt, Paris-Genève, Fallois-L'Âge d'Homme, 1989, p. 69

3 Franz [Ferenc] Liszt, Lettre à Odon Mihailovitch, cité par Serge Gut, Liszt, p. 432

4 Alexis de Tocqueville, Etat social et politique de la France avant et depuis 1789, dans Oeuvres complètes, tome VIII, Paris, Michel Lévy, 1865, pp. 47-48

5 Paul Bénichou, Le temps de prophètes, doctrines de l'âge romantique, Paris, Gallimard, 1977, p. 418

6 Bruno Moysan, Liszt, virtuose subversif, Lyon, Symétrie, 2010

7 Alexis de Tocqueville, Etat social et politique de la France, déjà cité

8 Norbert Elias, Mozart, sociologie d'un génie, Paris, Seuil, 1991 et particulièrement le chapitre «musicien bourgeois dans la société de cour», pp. 17-45

9 Alexis de Tocqueville, Etat social et politique de la France, déjà cité

10 Rémy Stricker, Franz Liszt ou les ténèbres de la gloire, Paris, Gallimard, 1993, p. 280

11 Stricker, Franz Liszt, p. 280

12 Sur ces fantaisies, voir notamment Bruno Moysan, La réécriture et ses enjeux dans les fantaisies de Liszt sur des thèmes d'opéra-1830-1848/Musique, sémantique, société, Lille, ANRT-Septentrion, 1998 dont une partie a été publiée dans Bruno Moysan, Liszt, virtuose subversif, Lyon, Symétrie, 2010

13 Marcel Gauchet, Philosophie des sciences historiques, Le moment romantique, Paris, Seuil, 2002, p. 11

14 Saint-Simon, Lettres d'un habitant de Genève à ses contemporains [1803] (Alfred Pereire ed.), Paris, Alcan, 1925, p. 25 